\title{
Shear viscous effects on the primordial power spectrum from warm inflation
}

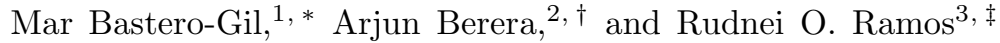 \\ ${ }^{1}$ Departamento de Fúsica Teórica y del Cosmos, \\ Universidad de Granada, Granada-18071, Spain \\ ${ }^{2}$ SUPA, School of Physics and Astronomy, University of Edinburgh, Edinburgh, EH9 3JZ, United Kingdom \\ ${ }^{3}$ Departamento de Física Teórica, Universidade do Estado do Rio de Janeiro, 20550-013 Rio de Janeiro, RJ, Brazil
}

\begin{abstract}
We compute the primordial curvature spectrum generated during warm inflation, including shear viscous effects. The primordial spectrum is dominated by the thermal fluctuations of the radiation bath, sourced by the dissipative term of the inflaton field. The dissipative coefficient $\Upsilon$, computed from first principles in the close-to-equilibrium approximation, depends in general on the temperature $T$, and this dependence renders the system of the linear fluctuations coupled. Whenever the dissipative coefficient is larger than the Hubble expansion rate $H$, there is a growing mode in the fluctuations before horizon crossing. However, dissipation intrinsically means departures from equilibrium, and therefore the presence of a shear viscous pressure in the radiation fluid. This in turn acts as an extra friction term for the radiation fluctuations that tends to damp the growth of the perturbations. Independently of the $T$ functional dependence of the dissipation and the shear viscosity, we find that when the shear viscous coefficient $\zeta_{s}$ is larger than $3 \rho_{r} / H$ at horizon crossing, $\rho_{r}$ being the radiation energy density, the shear damping effect wins and there is no growing mode in the spectrum.
\end{abstract}

PACS numbers: 98.80.Cq, 98.80.Es, 05.70.???a

\section{INTRODUCTION}

Cosmological observations, and in particular Cosmic Microwave Background (CMB) measurements [1-3], are consistent with a nearly gaussian and practically scale invariant spectrum of primordial perturbations, as predicted by the inflationary models [4, 5]. An early period of inflation also accounts for the inferred flatness of the Universe, and provides a solution to the horizon problem. This makes inflation a robust candidate to account for the early evolution of our universe. Inflationary predictions are characterized by the spectral index of the primordial spectrum, its tensor contribution, and the level of non-gaussianity. Present CMB data however sets at most an upper limit on the level of the tensor contribution and non-gaussianity [3] , and it is not yet able to discriminate among the different implementations and models of inflation. This situation is expected to change with the next generation of CMB experiments, like ESA's Planck surveyor satellite [6], which will further improve our knowledge of the cosmological parameters.

Inflation in brief is no more than an early period of accelerated expansion. In the standard picture of inflation, denoted as cold inflation, the universe rapidly supercools, and inflation should be followed by a reheating period, during which the inflationary vacuum energy is converted into radiation. For reheating to take place, the inflaton field has to couple to other degree of freedom, such that it can decay into light, relativistic degrees of freedom that thermalize and provide the radiation bath [7]. During cold inflation one assumes that those couplings play no role during the accelerated expansion. The alternative, called warm inflation [8, 9] (for earlier related work see [10]), assumes on the contrary that those coupling can lead to non-negligible dissipative effects, and radiation production can occur concurrently with the inflationary expansion. Both background evolution and inflaton fluctuations are modified with the inclusion of an extra friction term $\Upsilon \dot{\phi}$ accounting for the transfer of energy between the inflation and the radiation. The dynamics of the fluctuations are now governed by a Langevin equation including a noise force term from the influence of the radiation fluctuations into the inflaton field [8, 11 14]. Thermal fluctuations in the radiation are transfered to the inflaton and become the main source of primordial fluctuations [8, 12, 15].

*Electronic address: mbg@ugr.es

${ }^{\dagger}$ Electronic address: ab@ph.ed.ac.uk

‡Electronic address: rudnei@uerj.br 
The dissipative coefficient can be computed from first principles in quantum field theory, within an adiabatic approximation. The two-stage interaction configuration proposed in 13 has been shown to lead to a large enough dissipative coefficient while keeping the corrections to the inflationary potential under control and allowing a period of slow-roll inflation [16-18]. For the two-stage mechanism, the inflaton field couples to a heavy catalyst field, and the latter in turn couples to light degrees of freedom. During the motion of the background inflaton, it excites the catalyst fields which then decay into light fields [19]. Using this mechanism, the first calculation of the dissipative coefficient within the close-to-equilibrium approximation was done in [20], leading to a temperature dependent dissipative coefficient. In the low-temperature regime, when the mass of the heavy catalyst field is much larger than $T$, one has $\Upsilon \propto T^{3}$, while in the high-temperature regime, when $T$ is above the heavy catalyst field mass, depending on type of interaction the dissipative coefficient becomes linear with $T$ [21] or goes goes like the inverse of $T$ [22]. All high- $T$ models suffer in general from very large thermal corrections which spoil the flatness of the potential [22, 23], with only a few exceptions [12, 24]. However, viable models of warm inflation have been studied in the low- $T$ regime [25 27].

The temperature dependence of the dissipative coefficient induces the coupling of the field and radiation fluctuations as shown in 28]. Previous studies of the primordial spectrum of perturbations in warm inflation 15] did take into account the influence of the thermal fluctuations on the field through the noise term, but not the coupling through the dissipative term itself. In [28] it was shown that for positive power of $T$ in $\Upsilon$, and when $\Upsilon$ dominates over the Hubble expansion rate, this coupling induces a growing mode in the fluctuations before horizon crossing, enhancing by several order of magnitude the amplitude of the primordial perturbations with respect to previous calculations. In the calculations of the primordial spectrum typically the radiation bath is treated as a perfect fluid, with an equilibrium pressure $p_{r} \simeq \rho_{r} / 3$, where $\rho_{r}$ is the radiation energy density, an approximation valid in the close-to-equilibrium regime required for the calculation of the dissipative coefficient to hold. However, even in that regime, the radiation bath is expected to depart from an ideal fluid as a consequence of the constant production of radiation particles from the background field dissipation. Imperfect fluids have dissipative effects that can be parameterized in terms of shear and bulk viscosity coefficients, and a heat flow coefficient. Heat flow happens as a consequence of changes of conserved charges other than the stress-energy tensor, but we do not consider this possibility here and focus on the effects of the temperature. Bulk viscous effects, which can be interpreted as a consequence of the decay of particles within the fluid, have been considered for warm inflation in [29], where they studied either a constant bulk viscous pressure or one proportional to the radiation energy density. The bulk pressure appears at both the background and the perturbation level, and being a negative pressure, it will favor warm inflation. For the amplitude of the spectrum, for the phenomenological model considered in 29] they found that it could induce a variation in the amplitude of the order of $4 \%$. On the other hand, the shear viscosity is related to changes in momentum of the particles of the fluid, and appears only at the level of the perturbations. Shear and bulk viscosity coefficients due to light field have been extensively compute in the literature [30, 31], leading to power-law dependences on $T$ for these coefficients. In addition, the bulk viscous coefficient typically ends being much smaller than the shear viscosity. Therefore, we will concentrate on the effect of the shear viscosity on the spectrum, and do not consider those of the bulk viscosity. The shear viscosity, being related to dissipation, appears in the radiation fluid equation as a friction term which tends to damp the growth of the fluctuations [28]. Eventually, the damping effect will dominate over the enhancement induced by the dissipative source term. The aim will be therefore to quantify, in a model independent way, this effect on the spectrum, and when it will render the system field-radiation effectively decoupled.

The outline of the paper is as follow. In section II we review the basic of warm inflation at the background level. In section III we present the equations for the fluctuations of the coupled field-radiation system, when the radiation is taken as an imperfect fluid. The numerical solutions for the fluctuations are presented in section IV, with and without the shear viscosity. We also study the inflationary model dependence of the results on the spectrum by considering two generic model of inflation: a chaotic model with general power $p$, and a standard quadratic hybrid model. In section $\mathrm{V}$ we summarize our findings.

\section{WARM INFLATION: BACKGROUND EQUATIONS}

In any particle physics realization of the inflationary framework, the inflaton is not an isolated part of the model but it interacts with other fields. These interactions may lead to the dissipation of the inflaton energy into other degrees of freedom, such that a small percent of the inflaton vacuum energy is transferred into other kinds of energy. In the two-stage mechanism for warm inflation, dissipation leads to particle production of light degrees of freedom. When those relativistic particles thermalize fast enough, say in less than a Hubble time in 
an expanding universe, we can model their contribution as that of radiation:

$$
\rho_{r} \simeq \frac{\pi^{2}}{30} g_{*} T^{4},
$$

where $T$ is the temperature of the thermal bath, and $g_{*}$ the effective number of light degrees of freedom ${ }^{1}$.

The dissipative term appears as an extra friction term in the evolution equation for the inflaton field $\phi$,

$$
\ddot{\phi}+(3 H+\Upsilon) \dot{\phi}+V_{\phi}=0,
$$

$\Upsilon$ being the dissipative coefficient, $H=\dot{a} / a$ is the Hubble rate of expansion, and $a$ the scale factor of the Friedmann-Robertson-Walker background metric:

$$
d s^{2}=-d t^{2}+a(t)^{2} \delta_{i j} d x^{i} d x^{j} .
$$

Eq. (2.2) is equivalent to the evolution equation for the inflaton energy density $\rho_{\phi}$ :

$$
\dot{\rho}_{\phi}+3 H\left(\rho_{\phi}+p_{\phi}\right)=-\Upsilon\left(\rho_{\phi}+p_{\phi}\right),
$$

with pressure $p_{\phi}=\dot{\phi}^{2} / 2-V(\phi)$, and $\rho_{\phi}+p_{\phi}=\dot{\phi}^{2}$. Energy conservation then demands that the energy lost of the inflaton field must be gained by the radiation fluid $\rho_{r}$, with the RHS of Eq. (2.4) acting as the source term:

$$
\dot{\rho}_{r}+3 H\left(\rho_{r}+p_{r}\right)=\Upsilon\left(\rho_{\phi}+p_{\phi}\right) .
$$

In warm inflation, radiation is not redshifted away during inflation, because it is continuously fed by the inflaton through the dissipation [9]. Inflation happens when $\rho_{R} \ll \rho_{\phi}$, but even if small when compared to the inflaton energy density it can be larger than the expansion rate with $\rho_{R}^{1 / 4}>H$. Assuming thermalization, this translates roughly into $T>H$. Otherwise, when $T<H$ (or similarly when $\rho_{R}^{1 / 4}<H$ ), one just recovers the standard cold inflation scenario, where dissipation can be neglected.

During warm inflation the motion of the inflaton field has to be overdamped in order to have the accelerated expansion, but now this can be achieved due to the extra friction term $\Upsilon$ instead of that of the Hubble rate. And once $\phi, H$, and also $\Upsilon$, are in this slow-roll regime, the same will happen with the radiation energy density, the source term now compensating for the Hubble dilution. In the slow-roll regime, the equations of motion reduce to:

$$
\begin{aligned}
3 H(1+Q) \dot{\phi} & \simeq-V_{\phi}, \\
4 \rho_{R} & \simeq 3 Q \dot{\phi}^{2},
\end{aligned}
$$

where we have introduced the dissipative ratio $Q=\Upsilon /(3 H)$. Notice that $Q$ is not necessarily constant. The coefficient $\Upsilon$ will depend on $\phi$ and $T$, and therefore depending on the model the ratio $Q$ may increase or decrease during inflation [25]. The slow-roll conditions are given by [18]:

$$
\begin{aligned}
\epsilon & =\frac{m_{P}^{2}}{2}\left(\frac{V_{\phi}}{V}\right)^{2} \frac{1}{1+Q} \ll 1, \\
\eta & =m_{P}^{2}\left(\frac{V_{\phi \phi}}{V}\right) \frac{1}{1+Q} \ll 1, \\
\beta_{\Upsilon} & =m_{P}^{2}\left(\frac{\Upsilon_{\phi} V_{\phi}}{\Upsilon V}\right) \frac{1}{1+Q} \ll 1, \\
\delta & =\frac{T V_{T \phi}}{V_{\phi}}<1,
\end{aligned}
$$

\footnotetext{
${ }^{1}$ If not otherwise specified, we will take $g_{*}=228.75$, the effective no. of degrees of freedom for the Minimal Supersymmetric Standard Model, when presenting numerical results.
} 
where the slow-roll parameter $\beta_{\Upsilon}$ takes into account the variation of $\Upsilon$ with respect to $\phi$, and the last condition ensures that thermal corrections to the inflation potential are negligible. Similarly, taking also into account the dependence on $T$ of $\Upsilon$, one has:

$$
\left|\frac{d \ln \Upsilon}{d \ln T}\right|<4,
$$

which reflects the fact that radiation has to be produced at a rate larger than the redshift due to the expansion of the universe. The slow-roll regime ends when any of the above conditions Eqs. (2.8)-(2.11) is no longer satisfied, such that either the motion is no longer overdamped and slow-roll ends, or the radiation becomes comparable to the inflaton energy density. Either way, inflation will end shortly afterwards.

For warm inflation the first requirement is to have $T>H$, but the ratio $Q$ can be larger or smaller than unity. In the former case we are in the strong dissipative regime (SDR), whereas the latter is called weak dissipative regime (WDR). In the weak dissipative regime the extra friction added by $\Upsilon$ is not enough to substantially modify the background inflaton evolution, and it will resemble that of cold inflation; still the thermal fluctuations of the radiation energy density will modify the field fluctuations, and affect the primordial spectrum of perturbations.

The $T$ and $\phi$ dependent dissipative coefficient has been computed in [20], using the near-equilibrium approximation proposed in 22]. The specific field theory models considered for the inflaton interactions leading to dissipation all follow from the two-stage mechanism [13, 16, 32]. In this mechanism, the inflaton field $\phi$ is coupled to heavy catalyst fields $\chi$, which decay into light fields $\sigma_{i}$. Consistency of the approximations then demands the microphysical dynamics determining $\Upsilon$ to be faster than that of the macroscopic motion of the background inflaton and the expansion:

$$
\Gamma_{\chi}>\left|\frac{\dot{\phi}}{\phi}\right|, H,
$$

where $\Gamma_{\chi}$ is the decay width of the heavy fields. In addition, the condition $T \gg H$ allows to neglect the expansion of the universe when computing $\Upsilon$. In the low $T$ regime, when the mass of the catalyst field $m_{\chi}$ is larger than $T$, one has:

$$
\Upsilon(\phi, T) \propto \frac{T^{3}}{m_{\chi}^{2}} \propto \frac{T^{3}}{\phi^{2}},
$$

while in the high $T$ regime, where the thermal corrections to the catalyst field mass start to be important,

$$
\Upsilon(\phi, T) \propto T .
$$

And in the very high $T$ regime, the dissipative coefficient goes like the inverse of $T$. These are the cases of study we are going to consider in the next section when studying the fluctuations during warm inflation. In general, we will parametrize the dissipative coefficient as:

$$
\Upsilon=C_{\phi} \frac{T^{c}}{\phi^{m}},
$$

with $c-m=1$. We will work with $c=3,1,-1$, and also $c=0$, the case of no $T$ dependence for the dissipative coefficient.

\section{FLUCTUATIONS AT LINEAR ORDER: PRIMORDIAL SPECTRUM}

During warm inflation we have a multicomponent fluid, a mixture of a scalar inflaton field $\Phi$ interacting with the radiation fluid. Both components exchange energy and momentum through the dissipative term $\Upsilon$. Dissipative effects also imply small departures from equilibrium, and that the radiation fluid will not behave exactly like a perfect fluid during inflation. In relativistic theory, these effect can be parametrized in terms of a shear viscous tensor $\pi_{a b}$, an energy flux vector $q_{a}$ and a bulk viscous pressure $\pi_{b}$, in the stress-energy tensor for the radiation fluid [33, 34],

$$
T_{a b}^{(r)}=\left(\bar{\rho}_{r}+\bar{p}_{r}+\pi_{b}\right) u_{a}^{(r)} u_{b}^{(r)}+\left(\bar{p}_{r}+\pi_{b}\right) g_{a b}+q_{a}^{(r)} u_{b}^{(r)}+q_{b}^{(r)} u_{a}^{(r)}+\pi_{a b},
$$


where $\bar{\rho}_{r}$ is the energy density, $\bar{p}_{r}$ the adiabatic pressure, $u_{a}^{(r)}$ the four velocity of the radiation fluid, $g_{a b}$ the four-dimensional metric, and $u_{a}^{(r)} \pi^{a b}=0=g_{a b} \pi^{a b}, u_{a}^{(r)} q^{a}=0$. There would be heat flow for example in the presence of conserved charges in the system other than the stress-energy tensor, but we do not consider such possibility in this work, and then set $q_{a}=0$. The shear viscous tensor vanishes in an homogeneous and isotropic background geometry, but at linear order it is given by [34]:

$$
\pi_{a b} \simeq-2 \zeta_{s} \sigma_{a b},
$$

where $\zeta_{s}$ is the shear viscosity coefficient and $\sigma_{a b}$ the shear of the radiation fluid:

$$
\sigma_{a b}=\nabla_{(a} u_{b)}+u_{(a} u^{c} \nabla_{c} u_{b)}-\frac{h_{a b}}{3} \nabla^{c} u_{c},
$$

$\nabla_{a}$ being the covariant derivative of the metric $g_{a b}$. The bulk viscous pressure can be seen as a non-adiabatic pressure contribution, already present at the background level. Nevertheless, the contribution from the light fields is expected to be small with respect to $p_{r}$. Thus, we will also set $\pi_{b}=0$ and focus on studying the consequences of the shear viscosity on the primordial perturbations during warm inflation.

In order to study the system of perturbations at linear order, field, radiation energy density and radiation pressure are expanded around their background values in a Friedman-Robertson-Walker metric:

$$
\begin{gathered}
\Phi(x, t)=\phi(t)+\delta \phi(t, x), \\
\bar{\rho}_{r}(x, t)=\rho_{r}(t)+\delta \rho_{r}(t, x), \\
\bar{p}_{r}(x, t)=p_{r}(t)+\delta p_{r}(t, x),
\end{gathered}
$$

and similarly for the dissipative coefficient: $\bar{\Upsilon}(x, t)=\Upsilon(t)+\delta \Upsilon(t, x)$. The perturbed FRW metric, including only scalar perturbations, is given $b^{2}$ :

$$
d s^{2}=-(1+2 \alpha) d t^{2}-2 a \partial_{i} \beta d x^{i} d t+a^{2}\left[\delta_{i j}(1+2 \varphi)+2 \partial_{i} \partial_{j} \gamma\right] d x^{i} d x^{j},
$$

where $\varphi$ is the intrinsic curvature of a constant time hypersurface. For later use, we introduce the combinations:

$$
\begin{aligned}
& \chi=a(\beta+a \dot{\gamma}), \\
& \kappa=3(H \alpha-\dot{\varphi})+\partial_{k} \partial^{k} \chi,
\end{aligned}
$$

where $\chi$ is the shear and $-\kappa$ the perturbed expansion scalar of the comoving frame.

The evolution equations follow from the conservation of the energy-momentum tensor:

$$
\nabla^{a} T_{a b}^{(\alpha)}=Q_{b}^{(\alpha)}, \quad \sum_{\alpha} Q_{b}^{(\alpha)}=0,
$$

where $Q_{b}$ is the four-vector source term accounting for the exchange of energy and momentum:

$$
-Q_{b}^{(\phi)}=Q_{b}^{(r)}=\Upsilon u_{\phi}^{a} \nabla_{a} \Phi \nabla_{b} \Phi
$$

$u_{\phi}^{a}$ is now the four-velocity of inflaton fluid:

$$
u_{\phi}^{a}=-\frac{\nabla^{a} \Phi}{\sqrt{\rho_{\phi}+p_{\phi}}}
$$

and then:

$$
Q_{b}^{(r)}=\Upsilon\left(\bar{\rho}_{\phi}+\bar{p}_{\phi}\right)^{1 / 2} \nabla_{b} \Phi .
$$

The projection of the four-vector source term along the direction of the fluid gives the energy density source term,

$$
Q^{(r)}=-u_{\phi}^{a} Q_{a}^{(\phi)}
$$

\footnotetext{
${ }^{2}$ Latin indexes $i, j, k, \ldots$ are used for the spatial components, and either $a, b, c, \ldots$ or Greek letters for space-time indexes.
} 
which at linear order is given by:

$$
\begin{aligned}
Q^{(r)} & =Q_{r}+\delta Q_{r}, \\
Q_{r} & =\Upsilon \dot{\phi}^{2}, \\
\delta Q_{r} & =\delta \Upsilon \dot{\phi}^{2}+2 \Upsilon \dot{\phi} \delta \dot{\phi}-2 \alpha \Upsilon \dot{\phi}^{2} .
\end{aligned}
$$

The momentum source term $J_{a}$ is orthogonal to the fluid velocity:

$$
Q_{a}^{(\phi)}=Q^{(\phi)} u_{a}^{(\phi)}+J_{a}^{(\phi)}, \quad u^{(\phi) a} J_{a}^{(\phi)}=0,
$$

and vanishes in the FRW geometry; at linear order it reads:

$$
\begin{aligned}
J_{i}^{(r)} & =\partial_{i} \mathbf{J}_{r}, \\
\mathbf{J}_{r} & =-\Upsilon \dot{\phi} \delta \phi .
\end{aligned}
$$

To complete the specification of the source, we need $\delta \Upsilon$, which for a general temperature $T$ and field $\phi$ dependent dissipative coefficient, $\Upsilon=C_{\phi} T^{c} / \phi^{m}$, with $c-m=1$, is given by:

$$
\delta \Upsilon=\Upsilon\left(c \frac{\delta T}{T}-m \frac{\delta \phi}{\phi}\right) .
$$

Although dissipation implies departures from thermal equilibrium in the radiation fluid, the system has to be close-to-equilibrium for the calculation of the dissipative coefficient to hold, therefore $p_{r} \simeq \rho_{r} / 3, \rho_{r} \propto T^{4}$ and

$$
4 \frac{\delta T}{T} \simeq \frac{\delta \rho_{r}}{\rho_{r}} .
$$

Finally, the evolution equations for the radiation fluctuations are obtained expanding at linear order Eq. (3.10) 35 38]. Working in momentum space, defining the Fourier transform with respect to the comoving coordinates, the equation of motion for the fluctuations with comoving wavenumber $k$ are given by ${ }^{3}$ :

$$
\begin{aligned}
\delta \dot{\rho}_{r}+3 H\left(\delta \rho_{r}+\delta p_{r}\right) & =-3\left(\rho_{r}+p_{r}\right) \dot{\varphi}+\frac{k^{2}}{a^{2}}\left[\Psi_{r}+\left(\rho_{r}+p_{r}\right) \chi\right]+\delta Q_{r}+Q_{r} \alpha \\
\dot{\Psi}_{r}+3 H \Psi_{r} & =-\left(\rho_{r}+p_{r}\right) \alpha-\delta p_{r}+\frac{2 k^{2}}{3 a^{2}} \sigma_{r}+\mathbf{J}_{r}
\end{aligned}
$$

where a "dot" denotes the derivative with respect to the metric time " $t$ ", $\Psi_{r}$ is the radiation momentum perturbation, $T_{j}^{0(r)}=-\partial_{j} \Psi_{r} / a$, and $\sigma_{r}$ the shear viscous pressure at linear order:

$$
\sigma_{r} \simeq-2 \zeta_{s}\left(\frac{\Psi_{r}}{\rho_{r}+p_{r}}+\chi\right)
$$

On the other hand, field fluctuations are described by a stochastic system whose evolution is determined by a Langevin equation $[28$, 39] :

$$
\begin{aligned}
\delta \ddot{\phi}+(3 H+\Upsilon) \delta \dot{\phi}+\left(\frac{k^{2}}{a^{2}}+V_{\phi \phi}\right) \delta \phi & =[2(\Upsilon+H) T]^{1 / 2} a^{-3 / 2} \xi_{k}-\delta \Upsilon \dot{\phi} \\
& +\dot{\phi}(\kappa+\dot{\alpha})+(2 \ddot{\phi}+3 H \dot{\phi}) \alpha-\Upsilon(\delta \dot{\phi}-\alpha \dot{\phi}),
\end{aligned}
$$

where the stochastic source $\xi_{k}$ can be approximated by a localized gaussian distribution with correlation function:

$$
\left\langle\xi(t, x) \xi\left(t^{\prime}, x^{\prime}\right)\right\rangle=\delta\left(t-t^{\prime}\right) \delta^{(3)}\left(x-x^{\prime}\right) .
$$

\footnotetext{
${ }^{3}$ For simplicity, we keep the same notation for the fluctuations $\delta f(\mathbf{x}, t)$ and their Fourier transform $\delta f(\mathbf{k}, t)$.
} 
So far, the equations for the perturbations at linear order are written in a "gauge ready" form, without specifying any particular gauge, but the equations can also be written in terms of gauge invariant (GI) variables. For any scalar quantity $f$, at linear order one can define a gauge invariant perturbation [35, 36]:

$$
\delta f^{G I}=\delta f-\frac{\dot{f}}{H} \varphi,
$$

while similarly the gauge invariant momentum perturbation reads:

$$
\Psi^{G I}=\Psi-\frac{\rho+p}{H} \varphi
$$

and for the metric perturbations one has the gauge invariant combinations:

$$
\begin{aligned}
& \mathcal{A}=\alpha-\left[\frac{\varphi}{H}\right], \\
& \Phi=\varphi-H \chi .
\end{aligned}
$$

The evolution equations then read:

$$
\begin{aligned}
\delta \ddot{\phi}^{G I}+3 H \delta \dot{\phi}^{G I}+\left(\frac{k^{2}}{a^{2}}+V_{\phi \phi}\right) \delta \phi^{G I} & =[2(\Upsilon+H) T]^{1 / 2} a^{-3 / 2} \xi-\dot{\phi} \delta \Upsilon^{G I}-\Upsilon \delta \dot{\phi}^{G I} \\
& +\Upsilon \dot{\phi} \mathcal{A}+\dot{\phi} \dot{\mathcal{A}}+2(\ddot{\phi}+3 H \dot{\phi}) \mathcal{A}-\frac{k^{2}}{a^{2}} \dot{\phi} \frac{\Phi}{H}, \\
\delta \dot{\rho}_{r}^{G I}+3 H\left(1+w_{r}\right) \delta \rho_{r}^{G I} & =\frac{k^{2}}{a^{2}} \Psi_{r}^{G I}+\delta Q_{r}^{G I}+\dot{\rho}_{r} \mathcal{A}, \\
\dot{\Psi}_{r}^{G I}+3 H\left(1+\frac{k^{2}}{a^{2} H^{2}} \bar{\zeta}_{s}\right) \Psi_{r}^{G I} & =-w_{r} \delta \rho_{r}^{G I}-\Upsilon \dot{\phi} \delta \phi^{G I}-\left(\rho_{r}+p_{r}\right) \mathcal{A}-\frac{3 k^{2}}{a^{2}}\left(\rho_{r}+p_{r}\right) \bar{\zeta}_{s} \frac{\Phi}{H},
\end{aligned}
$$

where in Eq. (3.34) we have defined:

$$
\bar{\zeta}_{s}=\frac{4}{9} \frac{\zeta_{s} H}{\rho_{r}+p_{r}} .
$$

Finally, from the Einstein equations at linear order, the gauge invariant metric perturbations are given by [35, 36]:

$$
\begin{aligned}
\mathcal{A} & =-\frac{\dot{H}}{H^{2}} \mathcal{R}, \\
\frac{k^{2}}{a^{2} H^{2}} \Phi & =3 \mathcal{A}+\frac{3}{2} \frac{\delta \rho_{T}^{G I}}{\rho_{T}},
\end{aligned}
$$

where $\mathcal{R}$ is the total comoving curvature perturbation,

$$
\begin{aligned}
\mathcal{R} & =\varphi-\frac{H}{\rho_{T}+p_{T}} \Psi_{T} \\
& =-\frac{H}{\rho_{T}+p_{T}} \Psi_{T}^{G I} .
\end{aligned}
$$

For a multicomponent fluid, the total momentum perturbation is given by the sum of the individual components, and $\mathcal{R}$ can be written as the weighted sum of the individual contributions:

$$
\begin{aligned}
\mathcal{R} & =\sum_{\alpha=\phi, r} \frac{h_{\alpha}}{h_{T}} \mathcal{R}_{\alpha}, \\
\mathcal{R}_{\alpha} & =-\frac{H}{h_{\alpha}} \Psi_{\alpha}^{G I},
\end{aligned}
$$

where we have defined $h_{\alpha}=\rho_{\alpha}+p_{\alpha}$. In particular, for a scalar field $\Psi_{\phi}^{G I}=-\dot{\phi} \delta \phi^{G I}, h_{\phi}=\dot{\phi}^{2}$, and

$$
\mathcal{R}_{\phi}=\frac{H}{\dot{\phi}} \delta \phi^{G I},
$$


for which the power spectrum would be the form for single field cold inflation. For warm inflation, we shall use the total comoving curvature perturbation to evaluate the primordial spectrum,

$$
P_{\mathcal{R}}(k)=\frac{k^{3}}{2 \pi^{2}}\left\langle\left|\mathcal{R}_{k}\right|^{2}\right\rangle
$$

where " $\langle\cdots\rangle "$ means average over different realizations of the noise term in Eq. (3.26). In all numerical results shown in this work we have performed averages over 1000 runs. This was found to be more than enough to get convergent numerical results.".

The largest observable scale in the CMB corresponds to a comoving scale $k$ crossing the horizon $N_{e}$ e-folds before the end of inflation, denoted by $k=a_{*} H_{*}$. The value of $N_{e}$ can vary in general from 40 to 70 depending on the inflationary model and details on the subsequent reheating process [7]. Although we shall consider different inflationary potentials, we will not consider the details of reheating period, and simply fix the horizon crossing at $N_{e} \simeq 60$. As we will see, soon after horizon crossing, the amplitude of the individual curvature perturbations $\mathcal{R}_{\phi}$ and $\mathcal{R}_{r}$ freezes out, and so does that of the total curvature. This allows to compute the primordial spectrum by evaluating Eq. (3.42) at horizon crossing, mainly when getting analytical approximations. Nevertheless, when showing numerical results we shall integrate Eqs. (3.32)- (3.34) and evaluate the amplitude of the spectrum say 10 e-folds after horizon crossing.

\section{EQUATIONS IN THE ZERO-ORDER SLOW-ROLL APPROXIMATION AND BEYOND}

In order to gain some insight on the evolution of the perturbations, we follow 28] and first study the equations for the perturbations at zero-order in the slow-roll parameters. That is, expanding the background variables around their slow-roll values and neglecting all terms in the equations proportional to slow-roll parameters. This eliminates in the equations for the fluctuations the dependence on the inflationary potential, and the details on the evolution of the dissipative coefficient. For example, the metric perturbation $\mathcal{A}$ given in Eq. (3.36) is proportional to the slow-roll coefficient $\epsilon$, Eq. (2.8), $\epsilon=-\dot{H} / H^{2}$, and can be neglected; and the same for the terms proportional to $H \dot{\phi}$, i.e., those proportional to $\Phi$, and $\dot{\phi} / H \phi$. Defining the dimensionless variables:

$$
\begin{aligned}
y_{k} & =\frac{k^{3 / 2} \delta \phi^{G I}}{[2(\Upsilon+H) T]^{1 / 2}}, \\
w_{k} & =\frac{k^{3 / 2} \delta \rho_{r}^{G I}}{[2(\Upsilon+H) T]^{1 / 2}(\Upsilon \dot{\phi})}, \\
u_{k} & =\frac{k^{3 / 2} \Psi_{r}^{G I}}{[2(\Upsilon+H) T]^{1 / 2} \dot{\phi}},
\end{aligned}
$$

and using the slow-roll background equations (2.6), (2.7), we have the system:

$$
\begin{aligned}
\ddot{y}_{k}+3 H(1+Q) \dot{y}_{k}+H^{2}\left[z^{2}+3 \eta(1+Q)-3 m Q \frac{\dot{\phi}}{H \phi}\right] y_{k} & =\left(\frac{k}{a}\right)^{3 / 2} \xi_{k}-3 Q c H^{2} w_{k}, \\
\dot{w}_{k}+H(4-c) w_{k} & =\frac{H}{3 Q} z^{2} u_{k}+2 \dot{y}_{k}, \\
\dot{u}_{k}+3 H\left(1+z^{2} \bar{\zeta}_{s}\right) u_{k} & =-3 Q H\left(\frac{w_{k}}{3}+y_{k}\right),
\end{aligned}
$$

where $z=k /(a H)$. Combining Eqs. (4.5) and (4.6) into a second order differential equation, we have:

$$
\begin{aligned}
\ddot{w}_{k}+H\left(9-c+3 z^{2} \bar{\zeta}_{s}\right) \dot{w}_{k}+H^{2} & {\left[20-5 c+6 Q c+\frac{z^{2}}{3}+3 z^{2}(4-c) \bar{\zeta}_{s}\right] w_{k}=2\left(\frac{k}{a}\right)^{3 / 2} \xi } \\
+ & H\left(4-6 Q+6 z^{2} \bar{\zeta}_{s}\right) \dot{y}_{k}-H^{2}\left[3 z^{2}+6 \eta(1+Q)-6 Q m \frac{\dot{\phi}}{H \phi}\right] y_{k} .
\end{aligned}
$$




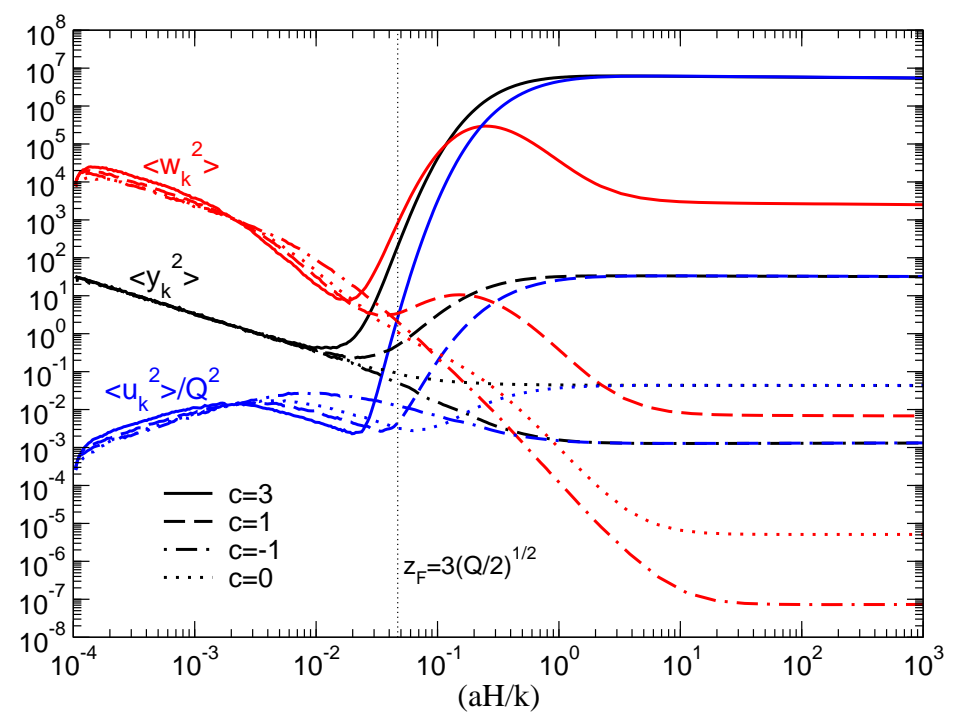

FIG. 1: Evolution of the power spectrum of the field $\left\langle y_{k}^{2}\right\rangle$, radiation energy density $\left\langle w_{k}^{2}\right\rangle$, and radiation momentum $\left\langle u_{k}^{2}\right\rangle / Q^{2}$, for $Q=100$, and wavenumber $k=10^{4} H$. The vertical thin dotted line sets the value of the freeze out scale $k_{F}$ in warm inflation. The results are shown for different power dependence on $T$ of the dissipative coefficient: $c=3$ (solid lines), $c=1$ (dashed lines), $c=-1$ (dash-dotted lines), and $c=0$ (dotted lines).

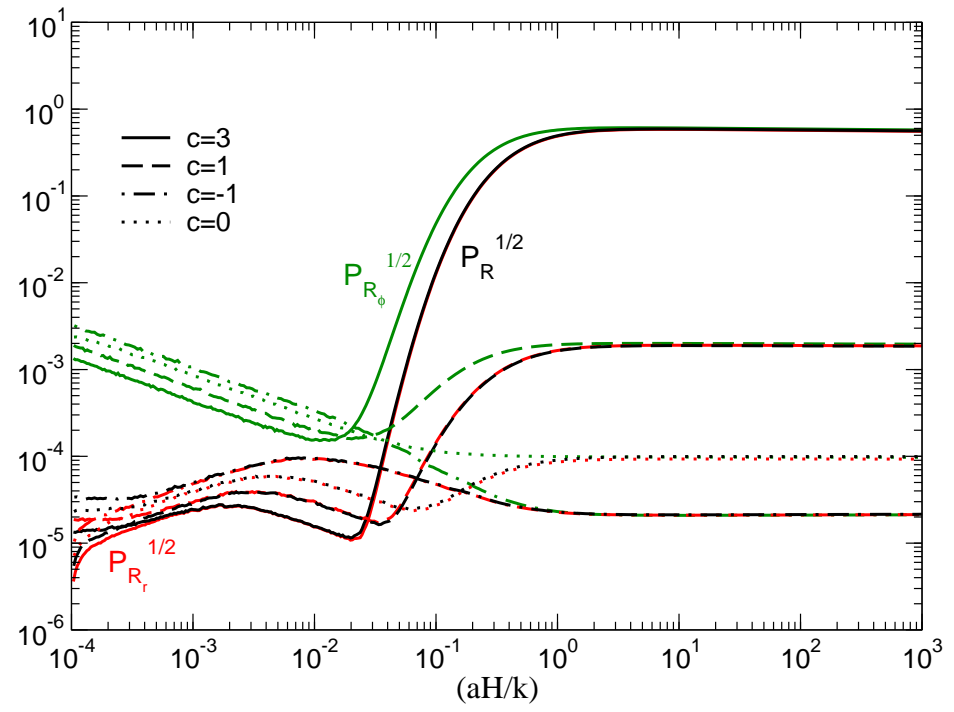

FIG. 2: Evolution of the total curvature perturbation spectrum $P_{\mathcal{R}}^{1 / 2}$ (black lines), the radiation $P_{\mathcal{R}_{r}}^{1 / 2}$ (red lines), and the field $P_{\mathcal{R}_{\phi}}^{1 / 2}$ (green lines) curvature perturbation spectrum. The results are shown for different power dependence on $T$ of the dissipative coefficient: $c=3$ (solid lines), $c=1$ (dashed lines), $c=-1$ (dash-dotted lines), and $c=0$ (dotted lines).

Before including shear effects, we study the evolution of the perturbations setting $\bar{\zeta}_{s}=0$. The evolution of the power spectrum of the field $\left\langle y_{k}^{2}\right\rangle$, the radiation energy density $\left\langle w_{k}^{2}\right\rangle$, and the radiation momentum $\left\langle u_{k}^{2}\right\rangle$, are shown in Fig. 1] as a function of $z^{-1}=a H / k$. We have taken $Q=100$, and started the integration at $z_{i}=10^{4}$. As mentioned above, quantities as $\left\langle y_{k}^{2}\right\rangle$ denotes the average over 1000 realizations of the gaussian noise term, and by $y_{k}^{2}, w_{k}^{2}, u_{k}^{2}$ we mean the modulus of the complex variable. We have set the initial conditions for field 
fluctuations in the vacuum, while $w_{k}$ and $u_{k}$ initially vanish, for simplicity. Starting the evolution early enough before horizon crossing, the system is always controlled by the stochastic source term, and the dependence on the initial conditions is quickly erased.

We have considered different powers of $T$ for the dissipative coefficient $\Upsilon, c=3,1,-1$, and included the case of a constant or field dependent $\Upsilon\left(c=0\right.$, dotted lines) for comparison. The radiation fluctuation $w_{k}$ acts as a source term for the field, but at early times $z^{-1} \ll 1$, for subhorizon perturbations, the field evolution is dominated by the stochastic source term, and both radiation and field fluctuations evolve like in the case $c=0$. In the latter case, the freeze out of the perturbations takes place before horizon crossing, due to the extra friction term in Eq. (4.4), at around $k_{F} /(a H) \simeq 3 \sqrt{Q / 2}$ (vertical thin dotted line) [12, 15, 28], and soon after field and radiation spectrum level off. The field spectrum for a $T$ independent $\Upsilon$ can be computed analytically and is given by [12]:

$$
\left\langle y_{k}^{2}\right\rangle_{0} \simeq \frac{\sqrt{3 \pi}}{4} \frac{\sqrt{1+Q}}{(1+3 Q)},
$$

where the subindex " 0 " denotes the value for $c=0$. On the other hand, when $c>0$, field and radiation fluctuations get effectively coupled before freeze out at around $z_{c}^{2} \approx 18 Q c$, and both start growing at similar rates. Numerically, we get:

$$
\left\langle y_{k}^{2}\right\rangle \approx\left\langle y_{k}^{2}\right\rangle_{z_{c}}\left(\frac{z_{c}}{z}\right)^{5 c}
$$

The field spectrum when the fluctuations are still subhorizon and $z>z_{c}$, which is independent of the radiation fluctuation, can be found in [28]:

$$
\left\langle y_{k}^{2}\right\rangle_{z_{c}} \approx \frac{3 c}{z_{c}}
$$

and therefore, at horizon crossing:

$$
\left\langle y_{k}^{2}\right\rangle_{*} \propto z_{c}^{5 c-1} \propto Q^{(5 c-1) / 2} .
$$

When $c<0$, the effect is the opposite, and effectively the freeze out is delayed until practically horizon crossing, which makes the amplitude of the field spectrum smaller than in the $c=0$ case.

Fig. 2] shows the evolution of the comoving curvature power spectrum, $P_{\mathcal{R}}^{1 / 2}$, given by the sum of the radiation and the field contributions as in Eq. (3.39), for different values of $c$. Also shown are the power spectra of the radiation, $P_{\mathcal{R}_{r}}^{1 / 2}$, and the field $P_{\mathcal{R}_{\phi}}^{1 / 2}$. After horizon crossing they all converge to the same amplitude. During slow-roll one has that $h_{r}=\rho_{r}+p_{r} \simeq Q h_{\phi}$, and from Eq. (4.6) when $z \ll 1$ the radiation momentum becomes proportional to the field fluctuation:

$$
\Psi_{r} \simeq Q \dot{\phi} \delta \phi
$$

and therefore:

$$
P_{\mathcal{R}_{r}}=\frac{H}{h_{r}} P_{\Psi_{r}} \simeq P_{\mathcal{R}_{\phi}} .
$$

Owing to the fact that $h_{\phi} \ll h_{r} \simeq h_{T}$, the main contribution to the primordial spectrum in Eq. (3.42) comes from the radiation, before and after horizon crossing. The primordial spectrum is always dominated by the thermal fluctuations. But after horizon crossing one simply has:

$$
P_{\mathcal{R}} \simeq P_{\mathcal{R}_{r}} \simeq P_{\mathcal{R}_{\phi}} .
$$

Therefore, the amplitude of the primordial spectrum can be written as usual in terms of that of the inflaton field:

$$
P_{\mathcal{R}} \simeq\left(\frac{H}{\dot{\phi}}\right)^{2} \frac{(H+\Upsilon) T}{\pi^{2}}\left\langle y_{k}^{2}\right\rangle_{*},
$$

evaluated at horizon crossing. 


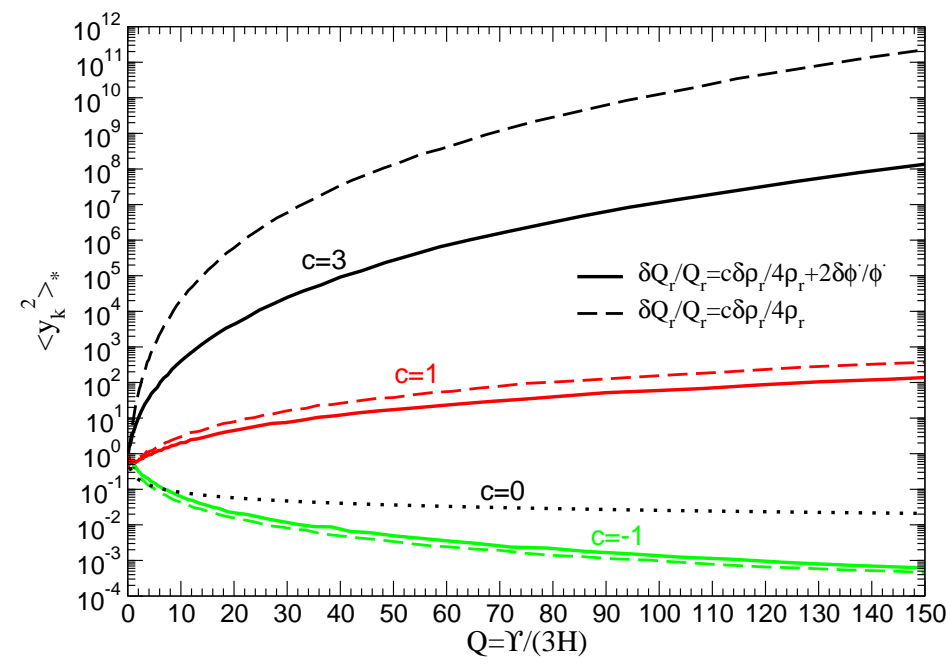

FIG. 3: The spectrum of the field at horizon crossing $\left\langle y_{k}^{2}\right\rangle_{*}$ as a function of the dissipative parameter $Q$, at zero order in the slow-roll parameters, for different values of $c$, and no shear $\bar{\zeta}_{s}=0$. Solid lines are obtained integrating Eqs. (4.4)-(4.6), while dashed lines were obtained with the approximation used in [28].

\begin{tabular}{|c|c|c|c|c|}
\hline$c$ & $\alpha$ & $\beta$ & $A_{c}$ & $B_{c}$ \\
\hline 3 & 7.5 & 7.0 & $1.9 \times 10^{-8}$ & $3.4 \times 10^{-6}$ \\
1 & 2.5 & 2.0 & $2.8 \times 10^{-2}$ & $6.8 \times 10^{-5}$ \\
-1 & 0.2 & 1.4 & 0.78 & 0.088 \\
\hline
\end{tabular}

TABLE I: Coefficients for the numerical fit of the spectrum, Eq. (4.16) and Eq. (4.17).

In Fig. 3 we have compared the power spectrum of the field, $\left\langle y_{k}^{2}\right\rangle$ at horizon crossing, as a function of the dissipative parameter $Q$ for different values of $c$. The equation for the fluctuations has been integrated keeping the background values constant (solid lines), Eqs. (4.4)-(4.6). The larger the power $c>0$, the larger the enhancement with $Q$, as the fluctuations get coupled earlier. For $c<0$, as mentioned before, we have the opposite effect, and the spectrum diminished with respect to the case $c=0$. For positive $c$, the curves can be fitted by a function:

$$
\left.\left\langle y_{k}^{2}\right\rangle_{*}\right|_{c>0} \simeq\left\langle y_{k}^{2}\right\rangle_{0}\left(A_{c} Q^{\alpha}+B_{c} Q^{\beta}\right) .
$$

but when $c=-1$, we have found that the curve can be best fitted by:

$$
\left.\left\langle y_{k}^{2}\right\rangle_{*}\right|_{c=-1} \simeq \frac{1+A_{-1} Q^{\alpha}}{1+B_{-1} Q^{\beta}} .
$$

The coefficients are given in Table \ For $c=3,1$, the approximation works well for $Q>50$, while for $c=-1$ it is valid for any $Q$.

We have also included in Fig. 3 the spectrum of the field obtained with the approximation used in 28 (dashed lines) for comparison. We have confirmed the main results about the power spectrum obtained in 28]; i.e., that for $c>0$, the amplitude of the primordial spectrum in warm inflation is enhanced by a factor $\simeq Q^{\alpha-1 / 2}$. However, while in [28] they found $\alpha=3 c$, we have a smaller power $\alpha=5 c / 2$, which for $c=3$ can mean a difference of two or 3 orders of magnitude in the amplitude of the spectrum for $Q \simeq 50-100$. The differences can be traced back to how the radiation source term is treated. In [28], it was approximated by:

$$
\delta Q_{r} \simeq Q_{r} \frac{\delta \Upsilon}{\Upsilon} \simeq c Q_{r} \frac{\delta \rho_{r}}{4 \rho_{r}},
$$

while we have kept the dependence on the field:

$$
\delta Q_{r} \simeq Q_{r}\left(c \frac{\delta \rho_{r}}{4 \rho_{r}}+2 \frac{\delta \dot{\phi}}{\dot{\phi}}\right) .
$$




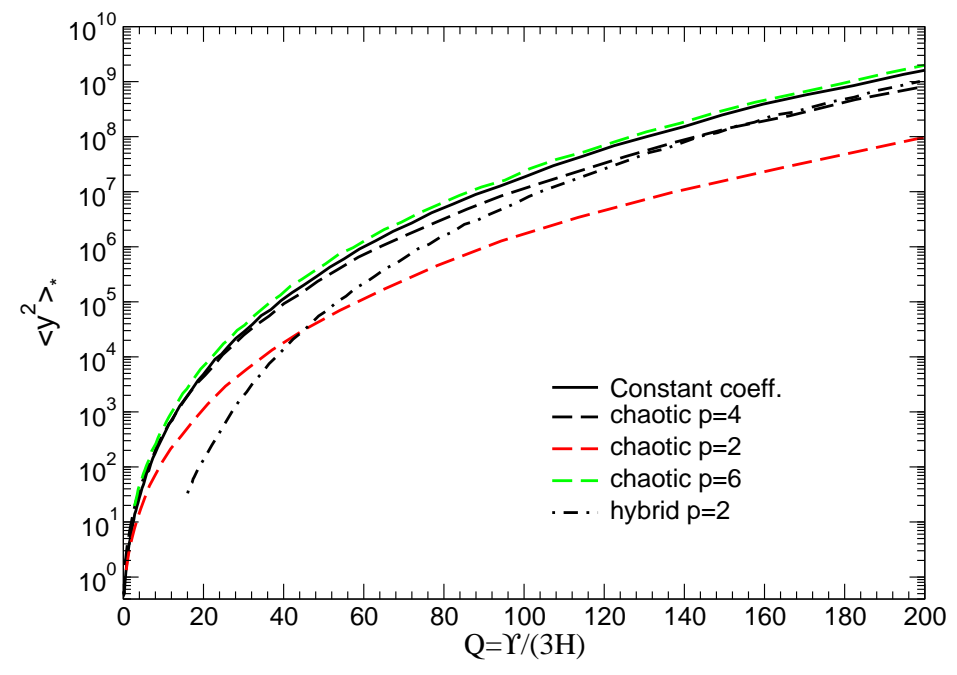

FIG. 4: The spectrum of the field $\left\langle y_{k}^{2}\right\rangle$ at horizon crossing as a function of the dissipative parameter $Q_{*}$, for different inflationary models, $c=3$ and no shear $\bar{\zeta}_{s}=0$. The solid line is the result at zero order in slow-roll; dashed lines are for a chaotic model with $p=6,4,2$, from top to bottom; the dash-dotted line is a quadratic hybrid model.

With no shear viscosity included, the second order differential equation Eq. (4.7) reads:

$$
\begin{aligned}
\ddot{w}_{k}+H(9-c) \dot{w}_{k}+H^{2} & \left(20-5 c \frac{z^{2}}{3}\right) w_{k}+H^{2} z^{2} y_{k}=2\left(\frac{k}{a}\right)^{3 / 2} \xi \\
& -H^{2} 6 Q c w_{k}+H(4-6 Q) \dot{y}_{k}-H^{2}\left[2 z^{2}+6 \eta(1+Q)-6 Q m \frac{\dot{\phi}}{H \phi}\right] y_{k},
\end{aligned}
$$

where we have written the equation such that on the RHS we have the terms induced by the field dependence in Eq. (4.19). Thus, setting the RHS to zero one recovers the equation derived with the source term as given in Eq. (4.18). While the term proportional to the field perturbation $y_{k}$ acts as a source term on the radiation that tends to enhance the fluctuation, the extra terms $6 H^{2} Q c w_{k}$ and $H(4-6 Q) \dot{y}_{k}$ have the opposite effect, i.e., that of damping the growth. Although these contributions are not enough to avoid the growth of the fluctuations, they have a sizable effect on their power-law behavior, mainly when $c=3$, as seen in Fig. 3. When $c=-1$ the radiation fluctuations do not grow before horizon crossing, so that the effect of the field dependent terms in Eq. (4.19) is negligible.

Neglecting the evolution of the background variables and working at zero order in the slow roll parameters, is a good approximation when $Q$ is large enough, and the background parameters hardly vary during the last 60 e-folds of inflation. Indeed the spectrum depends mainly on the value of the parameters in a smaller interval, 5-6 e-folds around horizon crossing, where one may expect the approximation of keeping them constant to be a fairly good one. Still, this is a model dependent question. This can be seen in Fig. 固 where we show the field spectrum for some generic inflationary models, and $c=3, \bar{\zeta}_{s}=0$. The value of $\left\langle y_{k}^{2}\right\rangle_{*}$ has been obtained integrating Eqs. (3.26)-(3.34), evaluating the amplitude of the comoving curvature spectrum at $N_{e}=20$ efolds after horizon crossing, and using Eq. (4.15). We have considered the inflationary models:

$$
\begin{array}{rlrl}
V & =\frac{V_{0}}{p}\left(\frac{\phi}{m_{P}}\right)^{p} & \text { (chaotic) }, \\
V & =V_{0}\left[1+\frac{\eta_{\phi}}{2}\left(\frac{\phi}{m_{P}}\right)^{2}\right], & & \text { (hybrid). }
\end{array}
$$

For the chaotic model we have run different powers $p=6,4,2$, and set $V_{0}=10^{-14} m_{P}^{4}$, while for the hybrid $V_{0}=10^{-8} m_{P}^{4}$ and $\eta_{\phi}=3$. For each model, the initial value of the inflation field is chosen such that we can have 


\begin{tabular}{|c|c|c|c|}
\hline$c$ & $A_{s}$ & $B_{s}$ & $\Delta_{s}$ \\
\hline 3 & 6.35 & 3.4 & 1.36 \\
1 & 1.9 & 1.2 & 1.33 \\
-1 & -0.95 & -0.7 & -0.66 \\
\hline
\end{tabular}

TABLE II: Coefficients for the numerical fit of the spectrum of the field with the shear viscosity, Eq. (4.23).

$N_{e} \simeq 64$, and from the background slow-roll equations one derives the initial values of the field derivative, $\rho_{r}$ and $Q$. We have chosen $k=100 H_{i}, H_{i}$ being the initial value of the Hubble parameter. Therefore, horizon crossing $k=a_{*} H_{*}$ takes places at around 60 e-folds before the end of inflation.

In all the examples considered above the dissipative coefficient increases during inflation [25], and the larger the power in the potential, the slower the evolution of the background values. In the plot, the solid line is the result obtained with constant background variables. For a quartic chaotic model or larger power, the approximation at zero order in the slow-roll works fine, while for a quadratic chaotic it tends to overestimate the spectrum by at least an order of magnitude for $Q \gtrsim 50$. For the hybrid model, the model dependence shows up when $Q \lesssim 100$.

Shear effects will further damp the growth of the fluctuations. In Eq. (4.7) the shear acts as an extra friction when the fluctuations are still subhorizon, suppressing the amplitude of the radiation fluctuation before the radiation-field system becomes effectively coupled. Whenever the shear is large enough, this suppression indeed can prevent altogether the growth of the field perturbations, as the amplitude of the radiation fluctuation is not enough to affect that of the field before horizon crossing. This will happen when $\bar{\zeta}_{s}=\zeta_{s} H /\left(3 \rho_{r}\right) \gtrsim 1$ at horizon crossing. During warm inflation, we have the catalyst field coupled to the inflaton field, and to the light degrees of freedom giving rise to the thermal bath. The shear viscosity for light fields (light with respect to the temperature $T$ of the thermal bath) typically behaves as $\zeta_{s} \propto T^{3}[31,40]$, although, depending on the pattern of interactions, other powers could be possible and cannot be excluded. Nevertheless, the damping is fully controlled by the value of the dimensionless parameter $\bar{\zeta}_{s}$ at horizon crossing, and is independent of the functional form of the shear with the temperature, as can be seen in Fig. 5. We have integrated the full EOM without approximations Eqs. (3.26)-3.34), for a quartic chaotic model, Eq. (4.21) with $p=4$, and set the initial background values such that $Q_{*} \simeq 40$. We show in the plot the dependence of the field spectrum with $\bar{\zeta}_{s}$ at horizon crossing, for different values of $c$, and two examples of the shear viscosity: one proportional to $T^{3}$ (solid lines), and another linear in $T$ (dashed lines), however the curves lay on top of each other. We have checked that this is independent of the inflationary model considered. We have normalized the field spectrum with the value obtained when $c=0$. As the shear increases, it does damp the radiation fluctuation enough for the field spectrum to approach the $c=0$ value. Asymptotically, when $\bar{\zeta}_{s} \gg 1$, for a linear dissipative coefficient with $T$ one practically recovers the $c=0$ case, for a cubic one the field spectrum is $\simeq 2\left\langle y_{k}^{2}\right\rangle_{0} / 5$, while the inverse $T$ case is slightly above $\simeq 5\left\langle y_{k}^{2}\right\rangle_{0} / 2$. In all cases, the field spectrum is well fitted by a function:

$$
\log _{10} \frac{\left\langle y_{k}^{2}\right\rangle}{\left\langle y_{k}^{2}\right\rangle_{0}} \simeq A_{s}-B_{s}\left[1+\tanh \left(\log _{10} \bar{\zeta}_{s}+\Delta_{s}\right)\right],
$$

which interpolates between the result with no shear $\sim 10^{A_{s}}$ and the $c=0$ case, modulo a normalization constant $\sim 10^{A_{s}-2 B_{s}}$. As an example, the coefficients $A_{s}, B_{s}$ and $\Delta_{s}$ for the potential shown in Fig. 5 with $Q_{*}=40$ are given in Table II]

Finally, combining Eq. (4.23) with Eq. (4.16), the field spectrum when $c>0$ reads:

$$
\left\langle y_{k}^{2}\right\rangle_{*} \simeq\left\langle y_{k}^{2}\right\rangle_{0} F_{Q}[Q]^{F_{s}\left[\bar{\zeta}_{s}\right]}
$$

where:

$$
\begin{aligned}
F_{Q}[Q] & \simeq\left(A_{c} Q^{\alpha}+B_{c} Q^{\beta}\right) \\
F_{s}\left[\bar{\zeta}_{s}\right] & \simeq \frac{1}{2}\left[1-\tanh \left(\log _{10} \bar{\zeta}_{s}+\Delta_{s}\right)\right] .
\end{aligned}
$$

Therefore, in the strong dissipative regime when $Q>1$, the primordial spectrum of the curvature perturbation can be written as that obtained for a $T$ independent dissipative coefficient, times an enhancement function $F_{Q}[Q]$ depending on the dissipative ratio $Q$, but controlled by a function depending on the shear $F_{s}\left[\bar{\zeta}_{s}\right]$ :

$$
P_{\mathcal{R}} \simeq\left(\frac{H^{2}}{\dot{\phi}}\right)^{2} \frac{\sqrt{3 \pi}}{4 \pi^{2}}\left[\frac{(1+Q)^{3 / 2}}{1+3 Q}\right]\left(\frac{T}{H}\right) \times F_{Q}[Q]^{F_{s}\left[\bar{\zeta}_{s}\right]},
$$




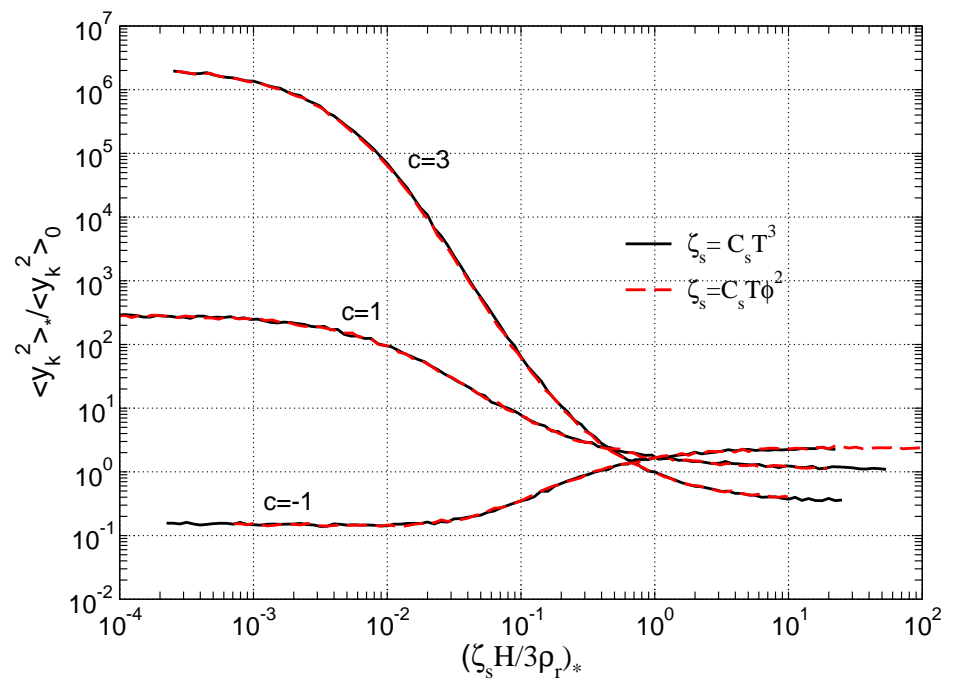

FIG. 5: Field spectrum normalized by the value with $c=0$, as a function of the shear parameter $\bar{\zeta}_{s}$, for different values of $c$, and $Q=40$. From top to bottom, $c=3,1,-1$. For each curve, we have also considered two different $T$ dependence on the shear viscosity, as indicated in the legend, but both gives the same field spectrum.

and whenever $\bar{\zeta}_{s}>1$ one recovers the amplitude of the primordial spectrum obtained when $c=0$. The latter is of a magnitude compatible with the observational value, for model parameters values common in inflationary model building [25, 26]. For example, without the enhancement, a quartic warm chaotic model gives rise to the right level of perturbations with a coupling constant $\lambda \simeq 10^{-13}-10^{-14}$. From the point of view of model building, the enhancement produced by the backreaction of the radiation fluctuations onto the fields, if not avoided by shear effects, can be compensated by lowering the height of the potential, i.e., by lowering couplings and masses. However, it will also have an impact on the prediction for the spectral index: for models with $Q$ increasing at the time of horizon crossing it will render the spectrum too blue-tilted, and the other way round, for $Q$ decreasing the spectrum may become too red-tilted. Shear viscosity damps the growth of the fluctuations, and therefore will also affect the spectral index.

Even though the results we have obtained are fairly model independent and shown not to depend on the specific dependence on the temperature of shear viscosity, it is useful to estimate the magnitude of the ratio $\zeta_{s} H /\left(3 \rho_{r}\right)$ for typical warm inflation models. In kinetic theory, the shear viscosity for relativistic fluids can be expressed parametrically as proportional to the mean free path of quasiparticles in the fluid. Considering as an example a radiation fluid made of relativistic scalar particles $\sigma$, with mass $m_{\sigma} / T \ll 1$ and self-interaction potential $\lambda_{\sigma} \sigma^{4} / 4$ !, the mean free path of quasiparticles is determined by the inverse of the thermal width, which is $\mathcal{O}\left(\lambda_{\sigma}^{2}\right)$, and the computed value for the shear viscosity is [30] $\zeta_{s} \simeq 3 \times 10^{3} T^{3} / \lambda_{\sigma}^{2}$. The condition $\zeta_{s} H /\left(3 \rho_{r}\right) \gtrsim 1$ can then be expressed, for example, as a condition on the magnitude of the radiation bath self-interaction, $\lambda_{\sigma} \lesssim 55 \sqrt{H / T}$. Since warm inflation requires $T>H$ and in general we typically work with values $T \gg H$, we find that weakly interacting radiation fluids can easily have shear viscosities of sufficient magnitude to counterbalance and suppress completely the growth of fluctuations caused by the coupling of the inflaton's fluctuations with those from the radiation.

In supersymmetric warm inflation models however the radiation bath self coupling is the same as the coupling to the catalyst field, which enters in the calculation of the dissipative coefficient. In general, large multiplicities of the fields (catalyst $\chi$ and radiation $\sigma$ ) are required in order to have enough dissipation. Considering a model with $\mathcal{N}_{\chi}, \mathcal{N}_{\sigma}$ copies of the complex fields, with common coupling $h$ among the $\chi$ 's and $\sigma$ 's, the self-interaction potential is given by $h^{2} \sum_{i}|\sigma|^{2} / 4$. In the low- $T$ regime, the dissipative and shear coefficients can be written as 


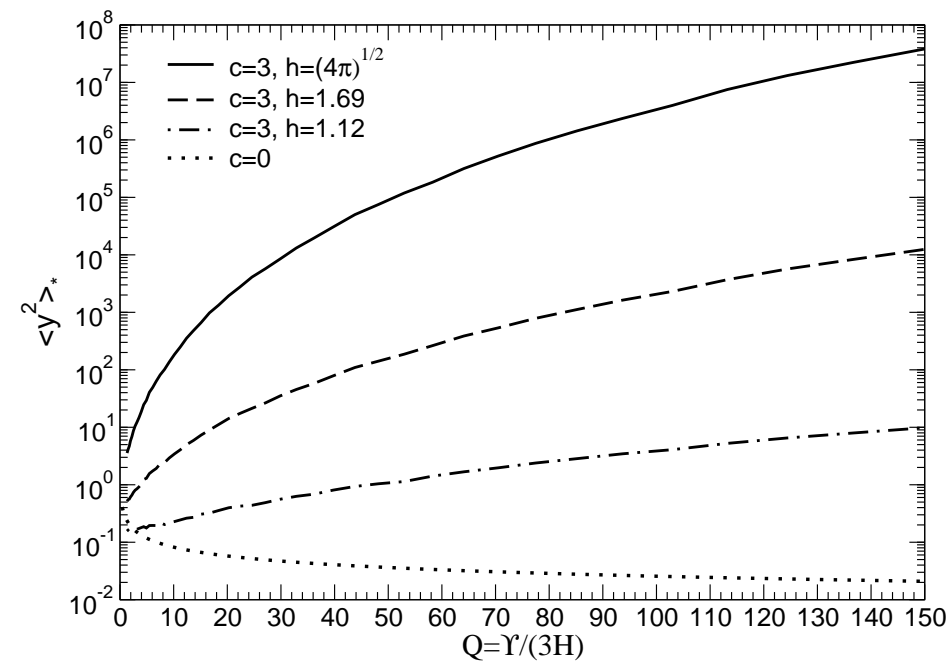

FIG. 6: Field spectrum as a function of $Q_{*}$, for a quartic chaotic model, with a cubic dissipative coefficient (Eq. 4.28) and $\zeta_{s}$ given by Eq. (4.29). We have taken $\mathcal{N}_{\sigma}=10^{3}$ and $h=\sqrt{4 \pi}$ (solid line), $h=1.67$ (dashed line) and $h=1.12$ (dot-dashed line). We include for comparison the result with $c=0$.

[21]:

$$
\begin{aligned}
& \Upsilon \simeq 0.1 h^{4} \mathcal{N}_{\chi} \mathcal{N}_{\sigma}^{2} \frac{T^{3}}{\phi^{2}}, \\
& \zeta_{s} \simeq 127 N_{\sigma}(1+0.3 h) \frac{T^{3}}{h^{4}},
\end{aligned}
$$

where we have included the next-to-leading order correction in the shear viscosity [40]. The condition $\bar{\zeta}_{s} \geq 1$ then reads:

$$
h^{4} \lesssim 128 \frac{\mathcal{N}_{\sigma}}{g_{*}} \frac{H}{T} \simeq 69 \frac{H}{T}
$$

where $g_{*} \simeq 15 \mathcal{N}_{\sigma} / 8$. Although in a bath of weakly interacting radiation particles, $h \ll 1$, the shear viscosity would be rather large, it may not give rise to enough dissipation to sustain a period of warm inflation, unless the weakness of the coupling is compensated by having a large multiplicity for the fields. This therefore becomes a model dependent question depending on the parameters $\mathcal{N}_{\chi}, \mathcal{N}_{\sigma}$ and the coupling $h$. In Fig. 6] we show an example for a quartic chaotic model, where we compare the value of the field spectrum $\left\langle y_{k}^{2}\right\rangle_{*}$ as a function of $Q_{*}$ for different values of the Yukawa coupling $h$. We have kept the value $\mathcal{N}_{\sigma}=10^{3}$ fixed, and vary the value of $\mathcal{N}_{\chi}$ to get different values of $Q_{*}$. From top to bottom the value of $h$ decreases, starting with the largest possible one $h=\sqrt{4 \pi}$ (solid line) for which there is a negligible shear effect. In this case, a value $Q_{*} \simeq 10$ requires $\mathcal{N}_{\chi}=35$, while for $Q_{*} \simeq 100$ we need $\mathcal{N}_{\chi} \simeq 110$. As the value of $h$ decreases, the multiplicity of the field to get the same value of $Q_{*}$ increases by a factor $\left(4 \pi / h^{2}\right)^{2}$. For $h=1.12$ and $Q_{*}=100$, we would need $\mathcal{N}_{\chi} \simeq 10^{4}$. As the value of $Q_{*}$ increases, so it does the ratio $T / H$, and therefore the parameter $\bar{\zeta}_{s}$ decreases along the curves. Although in this example viscous effects are not enough to completely avoid the growth of the perturbations, they bring it down to $\left\langle y_{k}^{2}\right\rangle_{*} \propto Q^{2.5}$ for $h=1,12$ and $\left\langle y_{k}^{2}\right\rangle_{*} \propto Q^{4.5}$ for $h=1.67$, instead of $\left\langle y_{k}^{2}\right\rangle_{*} \propto Q^{7}$.

\section{CONCLUSIONS}

Density perturbations in warm inflation are seeded by thermal fluctuations of the inflaton. In warm inflation the inflaton decays into radiation through a dissipation term in the inflaton's equation of motion and that originates from the microphysical interactions of the inflaton field with other degrees of freedom of the microscopic 
Lagrangian describing the complete system. The origin of the dissipation term and its quantum field theoretical treatment has been extensively discussed in the literature (for a recent review, see e.g. Ref. 19]). However, as radiation is produced during the inflaton's evolution, the full treatment of the spectrum of perturbations no longer involves only that of the inflaton's perturbations but must also account for radiation perturbations. This makes the treatment of density perturbations in warm inflation similar to a multifluid system. Since the larger is the dissipation the larger is expected to be the rate of radiation production, it becomes important the study of how the produced radiation and its perturbations backreacts on the inflaton's evolution and respective perturbations. In Ref. [28] it was shown that as a consequence of this backreaction, the infaton's perturbations can grow as the dissipation of the inflaton increases. This increasing of the inflaton's perturbations with increasing dissipation can, therefore, severely constrain the model parameters in warm inflation so as to cope with the known measured results for the CMB radiation. This backreaction of the produced radiation on the inflaton's perturbations is larger the stronger is the coupling between the radiation and the inflaton; in particular the larger the power of $T$ in the dissipative coefficient, the stronger the $T$ dependence on the perturbations. This is exemplified by the results shown in Fig. 3 ,

In this paper we have studied how this backreaction of the produced radiation, that can lead to this growth mode in the inflaton's perturbations, can be counterbalanced by the dissipative effects within the radiation fluid. Dissipative effects in the radiation fluid itself are described by viscosity terms. This is expected when the radiation fluid departs from equilibrium, which is the case in any dissipative system, where the produced radiation from the system does not immediately equilibrate in the radiation bath and its approach to equilibrium is controlled by viscosity coefficients, like the shear viscosity, the bulk viscosity and heat transport coefficients. We have focused on the dissipation effect as coming dominantly from a shear viscosity term in the fluctuation equations. We have then shown that the shear viscosity can effectively damp the radiation fluctuations so as to avoid altogether the appearance of the growth mode in the resulting perturbations. The results we have obtained are model independent and we have shown that the overall effect of compensation of the growth mode and its control is determined by the ratio $\zeta_{s} H /\left(3 \rho_{r}\right)$, where $\zeta_{s}$ is the shear viscosity coefficient, $H$ is the Hubble parameter and $\rho_{r}$ the radiation energy density. When the ratio is $\mathcal{O}(1)$ or larger, the growth mode disappears completely.

In this work we have only considered the coupling between the fluctuations in the inflaton field with those in the radiation through the (temperature dependence on the) dissipation coefficient in the inflaton's dynamics. But in a thermal bath, the parameters of the inflaton's potential can also adquire temperature corrections. Even though these thermal corrections can be kept under control and small in Supersymmetry model building realizations for warm inflation [13, 19, 25], they can still be large enough to provide extra sources of couplings between inflaton and radiation fluctuations and it should be interesting to analyze their effects in a future work. Likewise, there can be additional sources of dissipation in the radiation fluid, for example as coming from bulk viscosity, that can further help to damp any leftover growing modes as resulting from these additional couplings. In this work we have neglected the effects of the bulk viscosity on the grounds that it is in general much smaller than the shear viscosity. For example, for the self-interacting scalar field radiation discussed in section IV, the ratio of the bulk viscosity, $\zeta_{b}$, with the shear viscosity for a high temperature radiation fluid is $\zeta_{b} / \zeta_{s} \sim 10^{-9} \lambda_{\sigma}^{3}$, thus, it is negligible for a weakly interacting radiation bath. But there may be other interactions and energy regimes for the radiation fluid in which the bulk can be sizable and lead to effects in the density perturbation evolution (see e.g. Ref. [41]).

All these effects, starting with the shear viscosity, will also impact the second order evolution of the perturbations and thus the calculation of the non-gaussinity. Forthcoming cosmological data are expected very soon to set the level of non-gaussinity of the primordial spectrum, which clearly will help to discriminate among inflationary models. Warm inflation, being a type of multi-fluid model, falls into the category of models with a non-negligible value of the non-linearity parameter $f_{N L}$ for non-gaussinity. This parameter has been computed for a $T$ independent dissipative coefficient [42], and recently the $T$ dependence of $\Upsilon$ has been included [43], which provides an extra non-linear source in the field second order equation. However, if the coupling between field and radiation perturbations at first order is suppressed by viscous effects, qualitatively we expect the same to happen at second order. The question then is whether one simply recover the prediction for a constant dissipative coefficient, or non-linearities are further suppressed by viscous effects. These and other effects mentioned above will be studied elsewhere. 


\section{Acknowledgments}

A.B. acknowledges support from the STFC. R.O.R is partially supported by Conselho Nacional de Desenvolvimento Científico e Tecnológico $(\mathrm{CNPq}$ - Brasil). M.B.G. would like to thank the hospitality of the School of Physics and Astronomy at the University of Edinburgh which during her visit this work has started. MBG is partially supported by MICINN (FIS2010-17395) and "Junta de Andalucía" (FQM101), and by SUPA during the realization of this work in the UK.

[1] C. L. Bennet et al., Astrophys. J. 464 L1 (1996); K. M. Gorski et al., Astrophys. J. 464 L11 (1996)

[2] P. de Bernardis et al., Nature 404 (2000) 955; C. B. Netterfield et al., Astrophys. J. 571 (2002) 604; C. J. MacTavish et al., Astrophys. J. 647 (2006) 799; S. Hanany et al., Astrophys. J. 545 (2000) L5; R. Stompor, arXiv:astro-ph/0309409 A. Benoit, Astron. Astrophys. 399 (2003) L25.

[3] E. Komatsu et al. [ WMAP Collaboration ], Astrophys. J. Suppl. 192 (2011) 18 arXiv:1001.4538 [astro-ph.CO]]; D. Larson et al., Astrophys. J. Suppl. 192 (2011) 16 arXiv:1001.4635 [astro-ph.CO]].

[4] A. H. Guth, Phys. Rev. D 23 (1981) 347; K. Sato, Phys. Lett. B 99 (1981) 66; A. Albrecht and P. J. Steinhardt, Phys. Rev. Lett. 48 (1982) 1220; A. D. Linde, Phys. Lett. B 108 (1982) 389.

[5] A. D. Linde, Phys. Lett. B 129 (1983) 177.

[6] Planck Surveyor Mission: http://www.rssd.esa.int/Planck

[7] L. F. Abbott, E. Farhi, M. B. Wise, Phys. Lett. B117 (1982) 29; A. Albrecht, P. J. Steinhardt, M. S. Turner, F. Wilczek, Phys. Rev. Lett. 48 (1982) 1437; A. D. Dolgov, A. D. Linde, Phys. Lett. B116 (1982) 329.

[8] A. Berera and L. Z. Fang, Phys. Rev. Lett. 74 (1995) 1912 arXiv:astro-ph/9501024.

[9] A. Berera, Phys. Rev. Lett. 75 (1995) 3218; Phys. Rev. D 55 (1997) 3346.

[10] L. Z. Fang, Phys. Lett. B 95 (1980) 154 ; I. G. Moss, Phys. Lett. B 154 (1985) 12; J. Yokoyama and K. I. Maeda, Phys. Lett. B 207 (1988) 31.

[11] A. Berera, Phys. Rev. D 54 (1996) 2519 arXiv:hep-th/9601134.

[12] A. Berera, Nucl. Phys. B 585 (2000) 666 arXiv:hep-ph/9904409.

[13] A. Berera, R. O. Ramos, Phys. Rev. D63 (2001) 103509 arXiv:hep-ph/0101049].

[14] A. Berera, I. G. Moss, R. O. Ramos, Phys. Rev. D76 (2007) 083520 arXiv:0706.2793 [hep-ph]].

[15] A. N. Taylor, A. Berera, Phys. Rev. D62 (2000) 083517 arXiv:astro-ph/0006077; H. P. De Oliveira, S. E. Joras, Phys. Rev. D64 (2001) 063513 arXiv:gr-qc/0103089; H. P. De Oliveira, Phys. Lett. B526 (2002) 1 gr-qc/0202045]; L. M Hall, I. G. Moss, A. Berera, Phys. Rev. D69 (2004) 083525 arXiv:astro-ph/0305015; T. Matsuda, JCAP 0906 (2009) 002 arXiv:0905.0308 [astro-ph.CO]].

[16] A. Berera and R. O. Ramos, Phys. Rev. D 71, 023513 (2005); Phys. Lett. B 607 (2005) 1.

[17] L. M. H. Hall and I. G. Moss, Phys. Rev. D 71 (2005) 023514 arXiv:hep-ph/0408323.

[18] I. G. Moss and C. Xiong, JCAP 0811 (2008) 023 arXiv:0808.0261 [astro-ph]|.

[19] A. Berera, I. G. Moss and R. O. Ramos, Rept. Prog. Phys. 72 (2009) 026901 arXiv:0808.1855 [hep-ph]].

[20] I. G. Moss and C. Xiong, arXiv:hep-ph/0603266.

[21] M. Bastero-Gil, A. Berera, R. O. Ramos, arXiv:1008.1929 [hep-ph].

[22] A. Berera, M. Gleiser, R. O. Ramos, Phys. Rev. D58 (1998) 123508 arXiv:hep-ph/9803394 [hep-ph]].

[23] J. Yokoyama and A. D. Linde, Phys. Rev. D 60 (1999) 083509 arXiv:hep-ph/9809409.

[24] A. Berera, M. Gleiser, R. O. Ramos, Phys. Rev. Lett. 83 (1999) 264 arXiv:hep-ph/9809583.

[25] M. Bastero-Gil, A. Berera, Int. J. Mod. Phys. A24 2207 (2009) arXiv:0902.0521[hep-ph]].

[26] Y. Zhang, JCAP 0903 (2009) 023 arXiv:0903.0685 [hep-ph]].

[27] Y. -F. Cai, J. B. Dent, D. A. Easson, Phys. Rev. D83 (2011) 101301 arXiv:1011.4074 [hep-th]]; M. Bastero-Gil, A. Berera, J. G. Rosa, arXiv:1103.5623 [hep-th].

[28] C. Graham and I. G. Moss, JCAP 0907 (2009) 013 arXiv:0905.3500 [astro-ph.CO]].

[29] S. del Campo, R. Herrera, D. Pavon, J. R. Villanueva, JCAP 1008 (2010) 002 arXiv:1007.0103 [astro-ph.CO]]; S. del Campo, R. Herrera, D. Pavon, Phys. Rev. D75 (2007) 083518 astro-ph/0703604.

[30] S. Jeon, L. G. Yaffe, Phys. Rev. D53 (1996) 5799 hep-ph/9512263.

[31] G. Aarts, J. M. Martinez Resco, JHEP 0402 (2004) 061 hep-ph/0402192.

[32] A. Berera and R. O. Ramos, Phys. Lett. B567, 294 (2003) hep-ph/0308211].

[33] S. Weinberg, Gravitation and Cosmology, (New York, NY, Wiley, 1972).

[34] R. Maartens, Class. Quant. Grav. 12 (1995) 1455; R. Maartens, J. Triginer, Phys. Rev. D56 (1997) 4640 gr-qc/9707018; R. Maartens, arXiv:astro-ph/9609119.

[35] H. Kodama and M. Sasaki, Prog., Theor. Phys. Suppl. 78 (1984) 1.

[36] J.-C. Hwang, ApJ 375 (1991) 443.

[37] J. -c. Hwang, H. Noh, Class. Quant. Grav. 19 (2002) 527 astro-ph/0103244.

[38] K. A. Malik, D. Wands and C. Ungarelli, Phys. Rev. D 67 (2003) 063516 arXiv:astro-ph/0211602. 
[39] E. Calzetta and B. L. Hu, Phys. Rev. D37(1988) 2878.

[40] G. D. Moore, Phys. Rev. D76 (2007) 107702 arXiv:0706.3692 [hep-ph]].

[41] M. Giovannini, Class. Quant. Grav. 22 (2005) 5243.

[42] I. G. Moss and C. Xiong, JCAP 0704 (2007) 007 arXiv:astro-ph/0701302.

[43] I. G. Moss and T. Yeomans, arXiv:1102.2833 [astro-ph.CO]. 